Comment. Math. Helv. 74 (1999) 575-590

(C) 1999 Birkhäuser Verlag, Basel

$0010-2571 / 99 / 040575-16 \$ 1.50+0.20 / 0$

Commentarii Mathematici Helvetici

\title{
Simple singularities and topology of symplectically filling 4-manifold
}

\author{
Hiroshi Ohta* and Kaoru Ono**
}

\begin{abstract}
Topological restrictions of symplectically filling 4-manifolds of links around simple singularities are studied by using the Seiberg-Witten monopole equations. In particular, the intersection form of minimal symplectically filling 4-manifolds of the singularity of type $E_{8}$ is determined. Moreover, for the case of simply elliptic singularities, similar restrictions are obtained. In the proof, a vanishing theorem of the Seiberg-Witten invariant is discussed.
\end{abstract}

Mathematics Subject Classification (1991). Primary 57R57, 32S55, 57M50.

Keywords. Fillable contact structure, symplectic filling, simple singularity, simply elliptic singularity, the Seiberg-Witten monopole equations.

\section{Introduction}

Let $\Gamma$ be a finite subgroup of $S U(2)$. According to Klein's classification theorem, $\Gamma$ is classified by the Dynkin diagrams of type $A_{n}(n \geq 1), D_{n}(n \geq 4), E_{6}, E_{7}$ and $E_{8}$. The group $\Gamma$ acts isometrically on $\mathbf{C}^{2}$ and the quotient space $\mathbf{C}^{2} / \Gamma$ has only an isolated singularity at the origin. This is called a simple singularity or a rational double point. Let $\widetilde{\mathbf{C}^{2} / \Gamma}$ be the minimal resolution of $\mathbf{C}^{2} / \Gamma$. The exceptional set is a union of rational curves with self intersection number -2 . The configuration of the rational curves is described by the corresponding Dynkin diagram of $\Gamma$, in particular the intersection form can be represented by the negative definite Cartan matrix corresponding to the Dynkin diagram. It is also one of fundamental properties of simple singularities that the canonical bundle of the minimal resolution $\widetilde{\mathbf{C}^{2} / \Gamma}$ is trivial. On the other hand, we have another description of the quotient singularity by viewing it as an isolated hypersurface singularity. The ring of $\Gamma$-invariant polynomials on $\mathbf{C}^{2}$ is isomorphic to $\mathbf{C}[x, y, z] / R_{\Gamma}$, where

\footnotetext{
*Partially supported by Grant in-Aid for Scientific Research no 10640070, the Ministry of Education, Science, Sports and Culture, Japan.

** Partially supported by Grant in-Aid for Scientific Research no 11440015, the Ministry of Education, Science, Sports and Culture, Japan.
} 
$R_{\Gamma}$ is an ideal generated by a single polynomial $f_{\Gamma}(x, y, z)$ given by the following table. (See [B3] and [Sai2], for example).

\begin{tabular}{cc}
\hline Group $\Gamma$ & Polynomial $f_{\Gamma}(x, y, z)$ \\
\hline$A_{n}$ & $x^{n+1}+y^{2}+z^{2}$ \\
$D_{n}$ & $x^{2} y+y^{n-1}+z^{2}$ \\
$E_{6}$ & $x^{4}+y^{3}+z^{2}$ \\
$E_{7}$ & $x^{3} y+y^{3}+z^{2}$ \\
$E_{8}$ & $x^{5}+y^{3}+z^{2}$ \\
\hline
\end{tabular}

Then the quotient singularity can be seen at the origin in the hypersurface $\left\{(x, y, z) \in \mathbf{C}^{3} \mid f_{\Gamma}(x, y, z)=0\right\}$. Deforming the equation, so the complex structure, we have a non singular Kähler surface which is called the Milnor fibre [Mi], $M_{\Gamma}=\left\{(x, y, z) \in \mathbf{C}^{3} \mid f_{\Gamma}(x, y, z)=\epsilon\right\}$. Here $\epsilon$ is a nonzero number. The intersection form of $M_{\Gamma}$ is given by the Milnor lattice which is isomorphic to the corresponding negative definite Cartan matrix of $\Gamma$. Note that two non singular Kähler surfaces $\widetilde{\mathbf{C}^{2} / \Gamma}$ and $M_{\Gamma}$ are not isomorphic as complex manifolds. But an interesting theorem due to Brieskorn [B1][B2] shows that their underlying 4manifolds are diffeomorphic. This is a characteristic feature of simple singularities which is deeply related to existence of a simultaneous resolution. The main purpose of this paper is trying to understand this phenomena from the point of view of contact and symplectic geometry.

Let us consider the compact portions of the minimal resolution $\widetilde{\mathbf{C}^{2} / \Gamma}$ and the Milnor fibre $M_{\Gamma}$. Namely we consider $\widetilde{B^{4} / \Gamma}$ and $M_{\Gamma} \cap B^{6}$ where $B^{2 n}$ denotes the closed unit disk around the origin in $\mathbf{C}^{n}$. Of course, they have the same diffeomorphism type and the boundary is a rational homology 3 -sphere $S^{3} / \Gamma$. From the construction we have a specific contact structure $\xi_{0}$ on $S^{3} / \Gamma$. The contact structure $\xi_{0}$ is induced from a standard contact structure $\xi_{s t}$ on $S^{3}$ which is defined as follows. Viewing $S^{3}$ as a unit sphere in $\mathbf{C}^{2}$, we define the 2-plane field $\xi_{s t}$ on $S^{3}$ by the intersection of $T S^{3}$ and $J\left(T S^{3}\right)$. Here $J$ denotes the complex structure on $\mathbf{C}^{2}$. Then $\xi_{s t}$ is completely non integrable so defines a contact structure on $S^{3}$. The orientation on $S^{3} / \Gamma$ is induced from the complex structure $J$ on $\mathbf{C}^{2}$. Generally, for a contact structure $\xi$ on a 3-manifold $Y$, if there exists a symplectic 4-manifold $(X, \omega)$ such that the boundary of $X$ with symplectic orientation is $Y$ with the orientation coming from the contact structure and $\left.\omega\right|_{\xi}>0$, we call $\xi$ a weakly fillable contact structure and $(X, \omega)$ a weakly symplectically filling manifold of $(Y, \xi)$. Furthermore, if there exists a contact form $\theta$ for $\xi$ such that the restriction of $\omega$ to $Y$ equals to $d \theta$, we call $\xi$ a strongly fillable contact structure and $(X, \omega)$ a strongly symplectically filling manifold of $(Y, \xi)$. If $Y$ is a rational homology sphere, these notions coincide and we simply call them fillable contact structure and symplectically filling manifold respectively. Namely, we have 
Lemma 1.1. For a contact manifold $(Y, \xi)$ with a rational homology sphere $Y$, it is a strongly fillable structure if and only if it is a weakly filling structure.

Proof. It is obvious that a strong symplectically filling manifold is a weakly symplectically filling manifold. We shall show the converse. Since $Y$ is a rational homology sphere, the restriction of $\omega$ to a collar neighborhood $U \cong Y \times[0,1]$ is exact, i.e., $\left.\omega\right|_{U}=d \eta$, where $\eta$ is a 1 -form on $U$.

By Lemma 4.1 in [K-M2], we can choose a function $f$ on $[0,1)$ such that $\tilde{\omega}=$ $\omega+d(f \theta)$ is a symplectic form on $X-\partial X$, where $f$ denotes the pull back of the function $f$ on $[0,1)$ by the projection $U-\partial X \cong Y \times[0,1)$ to $[0,1)$ and $\theta$ a contact form for $\xi$.

Instead of $\tilde{\omega}$ above, we consider the following 2 -form, $\bar{\omega}=d(\rho \eta)+d(f \theta)$. Here $\rho$ is a function on $[0,1]$, which equals 1 on $\left[0, s_{0}\right]$ for some $s_{0} \in(0,1)$ and 0 near $s=1$, and considered as a function on $U$. Since the function $f$ in [K-M2] is monotone increasing and tends to $+\infty$ as $s$ tends to 1 , we can find a decreasing function $\rho$ with $0 \leq|\dot{\rho}|<<1 / \dot{f}$. This implies that $\bar{\omega}$ is non-degenerate and indeed a symplectic form proportional to $d \theta$ on $Y \times\left\{t_{0}\right\}$ for $t_{0}$ sufficiently close to 1 . By changing the coordinate $t$ by $s=f(t)$, the symplectic form $\bar{\omega}$ is written as $d(s \theta)$, which can be symplectically embedded into the symplectization of the contact manifold $Y$. In particular, $\left((X-U) \cup Y \times\left[0, t_{0}\right], \bar{\omega}\right)$ is a strong symplectic filling of $Y$.

Remark. The proof above implies that a symplectic form of a weakly symplectically filling of $(Y, \xi)$ can be modified to be a symplectic form of a strongly symplectically filling of it. This argument was used in $[\mathrm{E}]$.

By definition, $\xi_{s t}$ is a fillable contact structure and $\xi_{0}$ is a fillable contact structure on $S^{3} / \Gamma$ as well. In this paper we call $\xi_{0}$ the standard contact structure on $S^{3} / \Gamma$. Of course, as $\epsilon$ changes, the complex structure on the Milnor fibre $M_{\Gamma} \cap B^{6}$ changes. But we have a smooth family of contact structures parametrized by $\epsilon$ on the boundary $M_{\Gamma} \cap S^{5}$. Gray's theorem implies that these contact structures are isotopic. The contact structure on the boundary $M_{\Gamma} \cap S^{5}$ when $\epsilon=0$ is nothing but the contact structure on the boundary of the minimal resolution $\widetilde{B^{4} / \Gamma}$. Hence these contact structures are isotopic. We denoted the isotopic class by $\xi_{0}$ for simplicity. From this point of view, Brieskorn's theorem implies that there are two natural symplectically filling 4-manifolds $\widetilde{B^{4} / \Gamma}$ and $M_{\Gamma} \cap B^{6}$ for $\left(S^{3} / \Gamma, \xi_{0}\right)$ which are diffeomorphic but not isomorphic as complex manifolds. Speaking from the point of symplectic geometry, their symplectic structures can be joined by a smooth family of symplectic structures, using hyperKähler structure.

Now we can state our main theorems of this paper.

Theorem 1. Let $\Gamma$ be a finite subgroup of $S U(2)$ and $\left(S^{3} / \Gamma, \xi_{0}\right)$ be a rational homology 3-sphere with the standard contact structure. Then for any symplectically 
filling 4-manifold of $\left(S^{3} / \Gamma, \xi_{0}\right)$, the intersection form is negative definite.

Remark. If we take an another isolated hypersurface singularity instead of simple singularities and fix a fillable contact structure on the boundary of the neighborhood, we have symplectically filling 4-manifolds which have various types of topologies. For example (see also the paragraph before Theorem 4), we pick a polynomial $f(x, y, z)=x^{2}+y^{3}+z^{7}$ and denote by $R(2,3,7)$ the intersection with the minimal resolution of $\left\{(x, y, z) \in \mathbf{C}^{3} \mid f(x, y, z)=0\right\}$ at the origin and $B^{6}$. Let $N(2,3,7)$ be a compact portion of the Milnor fibre defined by $\left\{(x, y, z) \in \mathbf{C}^{3} \mid f(x, y, z)=\epsilon\right\} \cap B^{6}$. Then both $R(2,3,7)$ and $N(2,3,7)$ are symplectically filling 4-manifolds of the Brieskorn integral homology 3 -sphere $\Sigma(2,3,7)=\left\{(x, y, z) \in \mathbf{C}^{3} \mid f(x, y, z)=0\right\} \cap S^{5}$ with a fixed contact structure. Although $R(2,3,7)$ is negative definite, the intersection from of $N(2,3,7)$ is given by $E_{8} \oplus 2\left(\begin{array}{ll}0 & 1 \\ 1 & 0\end{array}\right)$, which is indefinite.

When $\Gamma$ is $E_{8}$ type, the quotient space $S^{3} / \Gamma$ is the Poincaré integral homology 3 -sphere. In this case we can obtain more exact description on topology of symplectically filling 4-manifold. We say a symplectic manifold $X$ is minimal, if $X$ contains no symplectically embedded two spheres of self intersection -1 .

Theorem 2. Let $\Gamma$ be a finite subgroup of $S U(2)$ of type $E_{8}$ and $\left(S^{3} / \Gamma, \xi_{0}\right)$ be the Poincaré integral homology 3-sphere with the standard contact structure. Then for any minimal symplectically filling 4-manifold of $\left(S^{3} / \Gamma, \xi_{0}\right)$, we have the following. (1). The canonical bundle is trivial.

(2). The intersection form is equivalent to the negative definite Cartan matrix of type $E_{8}$.

If we show (1), then the claim (2) follows as below. If the canonical bundle is trivial, the 4-manifold has a spin structure. Then from Froyshov's result $[\mathrm{F}]$ we have an upper bound estimate of the second Betti number of the 4-manifold. Moreover in the case $S^{3} / \Gamma$ is an integral homology 3 -sphere, the intersection form is unimodular. Therefore we can conclude the claim (2) in Theorem 2 by the Hasse-Minkowski classification theorem on unimodular quadratic forms. As for the other cases of $\Gamma$, we can also show the triviality of the canonical bundle of symplectically filling 4-manifold. But the proof is different from that for the case $E_{8}$ given in this paper, which is somewhat ad-hoc. For the general cases, the proof will be based on an extended version of Taubes' theorem [T2][T3], which says correspondence between $J$-holomorphic curves and monopole solutions on 4manifold with conical end. The details will be discussed in the forthcoming paper [K-O-O].

Furthermore by the argument similar to Theorem 1, we can also show the following. 
Theorem 3. Let $(X, \omega)$ be a symplectic 4-manifold with boundary. The boundary is a union $\bigsqcup_{i=1}^{N} S^{3} / \Gamma_{i}$. (Each $\Gamma_{i}$ may be different.) We take the standard contact structure on each boundary component $S^{3} / \Gamma_{i}$. If $(X, \omega)$ is a symplectically filling of $\bigsqcup_{i=1}^{N} S^{3} / \Gamma_{i}$, then $N$ must be equal to 1 . Namely the boundary must be connected.

In [M1], D. McDuff proved Theorem 3 when each $\Gamma_{i}$ is trivial. She also proved the connectedness of boundary for the case of lens space, but we note that the contact structure is different from ours. See the paragraph following Theorem 4 below.

Beyond the class of simple singularities, we next consider so called simply elliptic singularities $\tilde{E}_{6}, \tilde{E}_{7}$ and $\tilde{E}_{8}$ [Sai1]. These are hypersurface singularities defined by polynomials $f_{\tilde{E}_{6}}=x^{3}+y^{3}+z^{3}+a x y z,\left(a^{3}+27 \neq 0\right), f_{\tilde{E}_{7}}=x^{4}+y^{4}+z^{2}+$ axyz, $\left(a^{4}-64 \neq 0\right)$ and $f_{\tilde{E}_{8}}=x^{6}+y^{3}+z^{2}+a x y z,\left(a^{6}-432 \neq 0\right)$ respectively. The intersection form of the corresponding Milnor fibre is described by the extended Dynkin diagram $\tilde{E}_{6}, \tilde{E}_{7}$ and $\tilde{E}_{8}$ respectively. In particular, we have $b_{2}^{+}=0$ and $b_{2}^{0}=2$ in all cases, and $b_{2}^{-}=6,7,8$, according to the type of $\tilde{E}_{6}, \tilde{E}_{7}, \tilde{E}_{8}$. Here by $b_{2}^{*}$ we denote the number of $*$-eigen values of the intersection matrix. On the other hand, it is known that the minimal resolution can be identified with a total space of complex line bundle over $T^{2}$ with the first Chern class being $-3,-2$ and -1 respectively. Under this identification, the exceptional divisor is zero section with self-intersection $-3,-2$ and -1 . Thus, in the cases of simply elliptic singularities, the Milnor fibre has different topology from the minimal resolution. But the number of positive eigen values of the intersection form is zero in both cases. (From the description of the minimal resolution, we have a specific strongly fillable contact structure on the boundary defined by a connection 1-form. By the same argument as simple singularity cases the contact structure is isotopic to one on the boundary of the Milnor fibre.) In fact, we can show Theorem 4 as following.

Theorem 4. Let $\Sigma_{g}$ be a closed Riemann surface of genus $g$ and $Y(n, g) \rightarrow$ $\Sigma_{g}$ be an $S^{1}$-bundle over $\Sigma_{g}$ associated to a complex line bundle with the first Chern class $n<0$. We take a contact structure $\xi_{\text {conn }}$ on $Y(n, g)$ defined by a connection 1-form. This defines a strongly fillable contact structure. Suppose $n<$ $2-2 g$. Then for any strongly symplectically filling 4-manifold of $\left(Y(n, g), \xi_{\text {conn }}\right)$, the intersection form has no positive eigen value.

Note that $\left(Y(-1,0), \xi_{\text {conn }}\right)$ is $\left(S^{3}, \xi_{s t}\right)$ and $\left(Y(-2,0), \xi_{\text {conn }}\right)$ is $\left(S^{3} / \Gamma, \xi_{0}\right)$ with $\Gamma=A_{1}$. But when $n<-2$, the contact structure $\xi_{\text {conn }}$ on $Y(n, 0)$ is different from the standard contact structure $\xi_{0}$ on $S^{3} / \Gamma$ of type $A_{-n-1}$, In fact, we naturally have a disk bundle $D(n, 0) \rightarrow S^{2}$ associated to the complex line bundle with the first Chern class $n$, which is a strongly symplectically filling 4-manifold of $\left(Y(n, 0), \xi_{\text {conn }}\right)$, because $n<0$. The first Chern class of the complex tangent bundle of $D(n, 0)$ is $n+2$. Then the Euler class of the 2-plane field $\xi_{\text {conn }}$ is given by $(n+2)(\bmod n) \in H^{2}(Y(n, 0) ; \mathbf{Z})=\mathbf{Z} / n \mathbf{Z}$. So this cohomology class is non- 
trivial when $n \neq-2,-1$. On the other hand, for the case of $\left(S^{3} / \Gamma, \xi_{0}\right)$, the Euler class of contact 2-plane field $\xi_{0}$ is cohomologically trivial. Hence when $n<-2, \xi_{0}$ and $\xi_{\text {conn }}$ on $Y(n, 0)$ are different.

This work was presented at Symplectic Topology Workshop held at Fields Institute, Toronto, Workshop on Moment Maps and Quantization, and Workshop on Symplectic Topology at the University of Warwick. We are grateful to organizers of these conferences. In particular, during the Workshop on Symplectic Topology at Warwick, we learnt that P. Lisca also obtained related results independently.

\section{Proof of Theorem 1,3 and 4}

First of all, we shall prove Theorem 1. Let $\left(X_{\Gamma}, \omega\right)$ be a symplectically filling 4-manifold of $\left(S^{3} / \Gamma, \xi_{0}\right)$. First, by using a compactification of the Milnor fibre in certain weighted projective space, we shall symplectically glue $X_{\Gamma}$ along $\left(S^{3} / \Gamma, \xi_{0}\right)$ to obtain a symplectic closed 4-manifold.

Let $M_{\Gamma}$ be the Milnor fibre defined by $\left\{(x, y, z) \in \mathbf{C}^{3} \mid f_{\Gamma}(x, y, z)=\epsilon\right\}$. Here $f_{\Gamma}(x, y, z)$ is given by the table in the introduction. We regard $f_{\Gamma}(x, y, z)$ as a weighted homogeneous polynomial. The weights and degree are given by the following table according to the type of $\Gamma$.

\begin{tabular}{ccc}
\hline Group $\Gamma$ & Weights $(a, b, c)$ & degree $h$ \\
\hline$A_{n}$ & $\left(1, \frac{n+1}{2}, \frac{n+1}{2}\right)$ & $n+1$ \\
$D_{n}$ & $(n-2,2, n-1)$ & $2(n-1)$ \\
$E_{6}$ & $(3,4,6)$ & 12 \\
$E_{7}$ & $(4,6,9)$ & 18 \\
$E_{8}$ & $(6,10,15)$ & 30 \\
\hline
\end{tabular}

Consider a weighted projective space $\mathbf{P}(a, b, c, 1)=\left(\mathbf{C}^{4}-0\right) / \sim$, where $(x, y, z, w) \sim\left(t^{a} x, t^{b} y, t^{c} z, t w\right)$ for $t \in \mathbf{C}^{*}$. By a map $\iota$ from $\mathbf{C}^{3}$ to $\mathbf{P}(a, b, c, 1)$ defined by $\iota(x, y, z)=[x, y, z, 1], \mathbf{C}^{3}$ can be embeded as an open dense subset so that $\mathbf{P}(a, b, c, 1)=\mathbf{C}^{3} \cup \mathbf{P}(a, b, c)$. Here $\mathbf{P}(a, b, c)=\{[x, y, z, 0] \in \mathbf{P}(a, b, c, 1)\}$. We denote the closure of $\iota\left(M_{\Gamma}\right)$ in $\mathbf{P}(a, b, c, 1)$ by $\overline{M_{\Gamma}}$. It can be identified with a hypersurface $f_{\Gamma}(x, y, z)=w^{h}$ in the weighted projective space $\mathbf{P}(a, b, c, 1)$. The singular points on $\overline{M_{\Gamma}}$ lie on the complement $\overline{M_{\Gamma}}-\iota\left(M_{\Gamma}\right)=\overline{M_{\Gamma}} \cap \mathbf{P}(a, b, c)$. Taking the minimal resolution $\widetilde{M_{\Gamma}} \rightarrow \overline{M_{\Gamma}}$, we have a compact projective smooth surface $\widetilde{M_{\Gamma}}$. (See [Sai2]). This contains the Milnor fibre $M_{\Gamma}$ as an open dense subset.

We put $M_{\Gamma}^{\text {out }}=\widetilde{M_{\Gamma}}-\iota\left(M_{\Gamma} \cap B^{6}\right)$. Since $M_{\Gamma} \cap B^{6}$ is negative definite and $\widetilde{M_{\Gamma}}$ has a Kähler form, we have $b_{2}^{+}\left(M_{\Gamma}^{\text {out }}\right) \geq 1$. Moreover the boundary of $M_{\Gamma}^{\text {out }}$ is $S^{3} / \Gamma$. We put

$$
Z_{\Gamma}=X_{\Gamma} \cup_{S^{3} / \Gamma} M_{\Gamma}^{\text {out }} .
$$

Then we can show 
Lemma 2.1. $Z_{\Gamma}$ is a closed symplectic 4-manifold.

Proof. Take the singular point $p$ on $\overline{M_{\Gamma}} \subset \mathbf{C} P^{N}$ and a small standard ball $U$ centered at $p$ in $\mathbf{C} P^{N}$. We can modify the Fubini-Study form so that the restriction to $U$ is the flat standard Kähler form on the ball in $\mathbf{C}^{N}$. Then it is written as $d \lambda$ with $\lambda=\sqrt{-1} / 2 \sum\left(z_{j} d \bar{z}_{j}+\bar{z}_{j} d z_{j}\right)$. It is also a contact form for the contact structure given by maximally complex distribution on the boundary. Moreover, the symplectic form $d \lambda$ is isomorphic to the symplectic form on the symplectization of the contact manifold $\partial U$. Restricting $\lambda$ and $d \lambda$ to $\partial U \cap M_{\Gamma}$ and tubular neighborhood of $\partial U \cap M_{\Gamma}$ respectively, we get a symplectic embedding of a tubular neighborhood of $\partial U \cap M_{\Gamma}$ into the symplectization of $\partial U \cap M_{\Gamma}$, which is contactmorphic to $Y=S^{3} / \Gamma$.

On the other hand, Lemma 1.1 guarantees that the symplectic form of symplectically filling manifold can be modified so that its restriction to the collar neighborhood can be symplectically embedded into the symplectization of $(Y, \xi)$.

We fix a coorientation of $\xi$ and multiply -1 to the contact form if its coorientations is opposite. The contact form $\theta$ in Lemma 1.1 and the contact form $\lambda$ as above give sections of

$$
\operatorname{Symp}(Y):=\left\{\alpha \in T^{*} Y \mid \operatorname{Ker} \alpha=\xi\right\} .
$$

After multiplying sufficiently large number to $\lambda$, we may assume that these two sections do not intersect. We put $V:=\{\alpha \in \operatorname{Symp}(Y) \mid \theta \leq \alpha \leq \lambda\}$, where $\theta<\alpha$ means $\alpha-\theta$ gives the given coorientation of $\xi$. Then we glue $X_{\Gamma}, V$ and $M_{\Gamma}^{\text {out }}$ along boundaries, and get a smooth symplectic manifold $Z_{\Gamma}$.

Now suppose that $b_{2}^{+}\left(X_{\Gamma}\right) \geq 1$. Then we have $b_{2}^{+}\left(Z_{\Gamma}\right) \geq 2$. A theorem due to Taubes [T1] implies that the Seiberg-Witten invariant for the canonical Spin ${ }^{c}$ structure of the symplectic 4-manifold $Z_{\Gamma}$ is non trivial. On the other hand, we can show a vanishing theorem of Seiberg-Witten invariants as follows.

Proposition 2.2. Let $X$ be a closed oriented connected smooth 4-manifold. Suppose $X$ has a decomposition $X=X_{1} \cup_{Y} X_{2}$ so that $b_{2}^{+}\left(X_{i}\right) \geq 1,(i=1,2)$ and $Y$ is a closed oriented 3-manifold with a metric of positive scalar curvature. Then all the Seiberg-Witten invariants of $X$ vanish.

This might be a folk theorem which is well-known to specialists. In the section 4 , we shall prove it for convenience in the case $Y$ is a rational homology 3-sphere.

Since $S^{3} / \Gamma$ admits a metric of positive scalar curvature, by using the vanishing theorem, we have a contradiction if we suppose $b_{2}^{+}\left(X_{\Gamma}\right) \geq 1$. Hence we have proved Theorem 1. 3 .

By the argument similar to the proof of Theorem 1, we can also prove Theorem 
Proof of Theorem 3: Suppose $N \geq 2$. Using the outer part $M_{\Gamma_{i}}^{\text {out }}$ of the compactification of the Milnor fibre as in the proof of Theorem 1, we can construct a closed symplectic 4-manifold $Z_{\Gamma_{1}, \cdots, \Gamma_{N}}$ by

$$
Z_{\Gamma_{1}, \cdots, \Gamma_{N}}=X \cup \bigsqcup_{i=1}^{N} M_{\Gamma_{i}}^{\text {out }} .
$$

Since $b_{2}^{+}\left(M_{\Gamma_{i}}^{\text {out }}\right) \geq 1$, we find that all Seiberg-Witten invariants of $Z_{\Gamma_{1}, \cdots, \Gamma_{N}}$ vanish form Proposition 2.2, provided $N \geq 2$. This contradicts to Taubes' theorem [T1] that the Seiberg-Witten invariant for the canonical Spin ${ }^{c}$ structure of a symplectic 4-manifold with $b_{2}^{+} \geq 2$ is non-zero. Thus $N$ must be equal to 1 .

Finally, before ending this section, we prove Theorem 4 .

Proof of Theorem 4: Since $n<0$, we have a natural strongly filling symplectic manifold $(D(n, g), \omega)$ of $\left(Y(n, g), \xi_{\text {conn }}\right)$, which is a disk bundle over $\Sigma_{g}$ associated to the complex line bundle $L(n . g) \rightarrow \Sigma_{g}$ with the first Chern class $n$. We define a $\mathbf{C} P^{1}$-bundle $P(n, g) \rightarrow \Sigma_{g}$ to be the projectivization of $L(n, g)$ by $P(n, g)=$ $\mathbf{P}(L(n, g) \oplus \underline{\mathbf{C}})$, where $\underline{\mathbf{C}}$ is a trivial complex line bundle over $\Sigma_{g}$. In stead of the compactification of the Milnor fibre, we use $P(n, g) \rightarrow \Sigma_{g}$ which can be considered as a compactification of the minimal resolution. We denote by $\Sigma_{g}^{\infty}$ the section at infinity of $P(n, g)$. Note that the self-intersection number of $\Sigma_{g}^{\infty}$ is equal to $-n>0$. Naturally $D(n, g)$ is in $P(n, g)$. We put $P(n, g)^{\text {out }}=\overline{P(n, g)-D(n, g)}$. Now let $X$ be any strongly symplectically filling 4 -manifold of $\left(Y(n, g), \xi_{\text {conn }}\right)$. Then in a way similar to the proof of Theorem 1 , we can construct a closed symplectic 4-manifold $Z(n, g)$ by

$$
Z(n, g)=X \cup_{Y(n, g)} P(n, g)^{\text {out }} .
$$

Now suppose that $b_{2}^{+}(X) \geq 1$. Then $b_{2}^{+}(Z(n, g)) \geq 2$. Taubes' theorem [T3] shows the Poincaré dual of $c_{1}\left(K_{Z}\right)$ can be represented by a pseudo-holomorphic curve $C$. Since two distinct pseudo-holomorphic curves in a 4-manifold have non-negative intersection number [M2] and the self-intersection number of $\Sigma_{g}^{\infty}$ is positive, we have $C \cdot \Sigma_{g}^{\infty} \geq 0$. On the other hand, the adjunction formula for the pseudoholomorphic curve $\Sigma_{g}^{\infty}$ reads

$$
2 g-2=\left[\Sigma_{g}^{\infty}\right]^{2}+c_{1}\left(K_{Z}\right)\left[\Sigma_{g}^{\infty}\right]=-n+[C] \cdot\left[\Sigma_{g}^{\infty}\right] .
$$

If $n<2-2 g$, then we have $[C] \cdot\left[\Sigma_{g}^{\infty}\right]<0$, which is contradiction. Hence we have proved Theorem 4 . 


\section{Proof of Theorem 2}

Let $(X, \omega)$ be a minimal symplectically filling 4-manifold of $\left(S^{3} / \Gamma, \xi_{0}\right)$ with $\Gamma=$ $E_{8}$. As we mentioned in the introduction, it is enough to prove that the canonical bundle of $(X, \omega)$ is trivial. If we can show it, then by Froyshov's result $[\mathrm{F}]$ we have $b_{2}(X) \leq 8$. Here the right hand side is the rank of the Cartan matrix of type $E_{8}$. Then since the isomorphic class of negative definite unimodular quadratic forms over $\mathbf{Z}$ of even type with rank $\leq 8$ is only $E_{8}$, we obtain the conclusion of (2).

In the theory of K3 surfaces, it is known that there is a K3 surface $S$ such that its algebraic (Picard) lattice contains $E_{8}$. (See for example [B-P-V].) (Note that the intersection form of $\mathrm{K} 3$ surfaces is the direct sum of three copies of hyperbolic lattices and two copies of $E_{8}$ lattices.) It implies that this K3 surface $S$ contains 8 (-2)-rational curves whose dual graph is the Dynkin diagram of type $E_{8}$. After

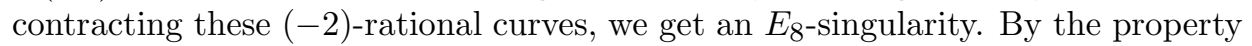
of $\mathrm{Du}$ Val singularities, this contracted space $W_{0}$ is a projective variety. (See, for example, Theorem 2.3 in [Art]).

In a similar way as in the proof of Lemma 2.1, we can make a closed 4-manifold $Z$ by gluing $X$ and $W=W_{0}-U$, where $U$ is a small open neighborhood of the $E_{8}$-singularity.

Since the intersection form $Q_{Z}$ of $Z$ is given by a direct sum $Q_{X} \oplus 3\left(\begin{array}{ll}0 & 1 \\ 1 & 0\end{array}\right) \oplus$ $E_{8}$ and $Q_{X}$ is negative definite from Theorem 1 , we have $b_{2}^{+}(Z)=3$. We can show in a way similar to Lemma 2.1 that

Lemma 3.1. $Z$ has a symplectic structure $\omega_{Z}$.

Proof. Note that $W_{0}$ is projective. The proof goes in a similar way as in the proof of Lemma 2.1.

Then it is enough to prove the canonical bundle $K_{Z}$ of $Z$ is trivial. We suppose that $K_{Z}$ is non trivial. Since $b_{2}^{+}(Z)>1$, Taubes' theorem [T1] shows the SeibergWitten invariant for the $\operatorname{Spin}^{c}$ structure with the characteristic line bundle $K_{Z}$ is non zero. Moreover thanks to Taubes's theorem [T2] [T3], the Poincaré dual of $c_{1}\left(K_{Z}\right)$ is represented by the fundamental class of an embedded symplectic curve $C$.

First we consider the case $C \cap W=\phi$. Namely the curve $C$ is contained in the interior of $X$. Since $X$ is negative definite, the self-intersection number of $C$ is negative. By dimension formula, the curve obtained by Taubes' theorem satisfies either that the curve is $(-1)$-curve or that the curve has non-negative intersection number. Hence the only possibility is a symplectic sphere with self-intersection -1 along which $X$ can be blown down. But we assumed $X$ is minimal. Hence this is a contradiction.

Next, we consider the case $C \cap W \neq \phi$. Since $C$ is a symplectic curve, we have 


$$
\int_{C \cap W} \omega_{Z}>0 .
$$

On the other hand, we can prove the following lemma which leads a contradiction. Thus we can prove $K_{Z}$ is trivial and so finish the proof of Theorem 2.

Lemma 3.2. Suppose $(X, \omega)$ is a strongly symplectically filling 4-manifold of $\left(S^{3} / E_{8}, \xi_{0}\right)$, that is, there is a 1-form $\lambda$ on $S^{3} / E_{8}$ such that $\operatorname{ker} \lambda=\xi_{0}$ and $\left.\omega\right|_{S^{3} / E_{8}}=d \lambda$. Then under the situation above we have

$$
\int_{C \cap W} \omega_{Z}<0
$$

Proof. Since $\left.K_{Z}\right|_{W}=\left.K_{S}\right|_{W}$ is trivial, $C \cap W$ is homologous to zero in $W$ relative to $\partial W$. Thus the $C \cap W$ is homologous to a 2-relative cycle $D$, contained in $\partial W$, with boundary $\partial(C \cap W)$. So by Stokes' theorem we have

$$
\int_{C \cap W} \omega_{Z}=\int_{D} d \lambda=\int_{\partial(C \cap W)} \lambda .
$$

We shall show the last quantity is negative.

Since $X$ is a strongly symplectic filling manifold, we have a Liouville vector field $\nu$ near $\partial W$, i.e., $\mathcal{L}_{\nu} \omega=\omega$ and $\nu$ is transversal to $\partial W$. ( $\nu$ is pointing interior of $W$.) Then we have $\lambda=i(\nu) \omega$. We may assume the intersection $\partial(C \cap W)$ is a union of embedded circles by perturbing the boundary $\partial W$. The orientation of $C$ as a holomorphic curve induces an orientation on $\partial(C \cap W)$. We denote by $v$ the vector field along $\partial(C \cap W)$, which is positive with respect to the boundary orientation. In particular, $J v$ is pointing interior of $W$. Then $\lambda(v)=i(\nu) \omega(v)=$ $\omega(\nu, v)=g_{J}(\nu,-J v)$, which is negative.

\section{Vanishing Theorem}

In this section we shall prove Proposition 2.2 for the case $Y$ is a rational homology 3 -sphere with a metric of positive scalar curvature. The assumption that $Y$ is a rational homology 3 -sphere is not necessary. But we assume it, because the vanishing theorem is known as a folklore and we like to avoid here the longer proof for the general case. This section is written in somewhat expository manner. 


\subsection{Monopole equations on 3-manifold}

Let $Y$ be a closed oriented connected 3-manifold with a Riemannian metric. Pick a $\operatorname{Spin}^{c}$ structure $t$ on $Y$ and a real valued closed 2-form $\mu$ on $Y$. Let $W$ be the Hermitian $\mathbf{C}^{2}$-bundle on $Y$ associated to the $\operatorname{Spin}^{c}(3)$ bundle corresponding to the $\operatorname{Spin}^{c}$ structure $t$. We denote by $\mathcal{A}(t)$ the $L_{1}^{2}$-completion of the set of spin connections on $Y$. Following [K-M2], we define the $\mu$-perturbed Chern-SimonsDirac functional $C S D_{\mu}$ on $\mathcal{A}(t) \times \Gamma(W)$ by

$$
C S D_{\mu}(B, \Psi)=-\frac{1}{2} \int_{Y}\left(B-B_{0}\right) \wedge\left(F_{\hat{B}}+F_{\hat{B}_{0}}+2 i \mu\right)-\int_{Y}<\Psi, D_{B} \Psi>.
$$

Here $B_{0}$ is a base point connection in $\mathcal{A}(t), F_{\hat{B}}$ is the curvature form of the connection $\hat{B}$ on the determinant line bundle of $W$ induced by $B$ and the LeviCivita connection and $D_{B}$ is the Dirac operator on $Y$. We denote by $\mathcal{G}_{Y}$ the $L_{2}^{2}$-completion of the gauge group $\operatorname{Map}(Y, U(1))$. For $u \in \mathcal{G}_{Y}$ we have

$$
C S D_{\mu}\left(B-u^{-1} d u, u \Psi\right)=C S D_{\mu}(B, \Psi)+\left(-4 \pi^{2} c_{1}(W)+2 \pi[\mu]\right) \cup[u],
$$

where $[u]$ is regarded as an element of $H^{1}(Y, \mathbf{Z})=[Y, U(1)]$ defined by the homotopy class of $u$. In temporal gauge, the (perturbed) monopole equations for the pull back $\operatorname{Spin}^{c}$ structure on $\mathbf{R} \times Y$ with the product metric can be interpreted as the gradient flow equations for the functional $C S D_{\mu}$. The set of critical points of $C S D_{\mu}$ are solutions to the equations

$$
\begin{aligned}
\rho\left(F_{\hat{B}}+i \mu\right)-\frac{1}{2}\left\{\Psi \otimes \Psi^{*}\right\} & =0 \\
D_{B} \Psi & =0 .
\end{aligned}
$$

As for notations used here, we refer to [K-M2]. We denote by $N_{\mu}(Y, t)$ the set of gauge equivalence classes of solutions to these equations. We say a solution $(B, \Psi)$ is reducible if $\Psi \equiv 0$ and irreducible if not.

Now we assume $Y$ is a rational homology 3 -sphere. In this case $C S D_{\mu}$ is invariant under $\mathcal{G}_{Y}$ by (1) and there is a unique reducible solution $(\theta, 0)$ for any $\operatorname{Spin}^{c}$ structure. Then Froyshov's result $[\mathrm{F}]$ shows

Fact A. Assume that $Y$ is a rational homology 3-sphere. Then there is a Baire set of exact $C^{r}$ 2-forms $\mu$ for which all points, including reducible solutions, in $N_{\mu}(Y, s)$ are non-degenerate.

Moreover suppose $Y$ admits a metric of positive scalar curvature. Then taking $\mu$ as in Fact A with the $C^{0}$ norm being sufficiently small, there is no irreducible solution. This follows from the usual boundedness argument of the $C^{0}$ norm of a solution $\Psi$ above from the norm of $\mu$ and the minus scalar curvature. (See [K-M1], $[\mathrm{F}])$. Therefore if we choose $\mu$ as above, the set $N_{\mu}(Y, t)$ consists of a single point $(\theta, 0)$, which is reducible and non-degenerate. 


\subsection{4-manifold with a cylindrical end}

From now on, we assume $Y$ is a rational homology 3 -sphere and admits a metric of positive scalar curvature. Let $\hat{X}$ be an oriented Riemanian 4-manifold with a cylindrical end $[-1, \infty) \times Y$. Fix a $\operatorname{Spin}^{c}$ structure $s$ on $\hat{X}$ which induces a $\operatorname{Spin}^{c}$ structure $t$ on $Y$. We choose and fix an exact 2-form $\mu$ on $Y$ such that the unique solution $(\theta, 0)$ in $N_{\mu}(Y, t)$ is non-degenerate as in 4.1. Let $\left(A_{\theta}, \Phi_{0}\right)$ be a spin connection and a spinor on $\hat{X}$ such that they are gauge equivalent to the pull back of $(\theta, 0)$ on the end of $X$. Since $(\theta, 0)$ is non-degenerate, the eigenvales of the Hessian of $C S D_{\mu}$ at $(\theta, 0)$ do not cluster at 0 . Thus we denote by $\epsilon_{0}>0$ the least positive eigenvalue of the Hessian. Take any positive number $\epsilon$ with $\epsilon<\epsilon_{0}$. Let $\pi:[-1, \infty) \times Y \rightarrow Y$ be the projection. We put

$$
\begin{aligned}
\mathcal{C}_{\hat{X}} & =\left\{(A, \Phi) \mid\left(A-A_{\theta}\right) \in e^{-\epsilon \tau} L_{1}^{2}, \text { and }\left(\Phi-\Phi_{0}\right) \in e^{-\epsilon \tau} L_{1, A_{0}}^{2}\right\} \\
\mathcal{G}_{\hat{X}} & =\left\{g \in \operatorname{Map}(\hat{X}, U(1)) \mid g-\pi^{*} g_{0} \in e^{-\epsilon \tau} L_{2}^{2}\right. \\
& \text { on the end for some } \left.g_{0} \in I_{(\theta, 0)}\right\}
\end{aligned}
$$

Here $\tau$ is a real valued function on $\hat{X}$ which agrees the coordinate in the direction $[-1, \infty)$ on the cylinder $[-1, \infty) \times Y$ and $I_{(\theta, 0)}$ is a stabilizer of $(\theta, 0)$ in $\mathcal{G}_{Y}$ which is isomorphic to $U(1)$. Let $\beta: \hat{X} \rightarrow \mathbf{R}$ be a non-negative cutoff function supported in the cylinder $[-1, \infty) \times Y$ and equal to 1 on $[0, \infty) \times Y$. We choose a real valued self-dual 2-form $\eta$ on $\hat{X}$ whose support is contained in a compact subset of $\hat{X}-[-1, \infty) \times Y$. Under the preparation above, we define $\mathcal{M}_{\eta, \mu}(\hat{X}, s)$ by the set of $\mathcal{G}_{\hat{X}}$-equivalence classes of solutions $(A, \Phi) \in \mathcal{C}_{\hat{X}}$ to the equations

$$
\begin{aligned}
\rho\left(F_{A}^{+}+i\left(\eta+\left(\beta \pi^{*} \mu\right)^{+}\right)\right)-\left\{\Phi \otimes \Phi^{*}\right\} & =0 \\
D_{A}^{+} \Phi & =0 .
\end{aligned}
$$

Here $\left(\beta \pi^{*} \mu\right)^{+}$is the self-dual part of $\beta \pi^{*} \mu$ Note that $\mathcal{M}_{\eta, \mu}(\hat{X}, s)$ does not depend on the choice of $\left(A_{\theta}, \Phi_{0}\right)$ because $H^{1}(Y, \mathbf{Z})=0$. (See [K-M2] 5.(iii)). We call a solution $(A, \Phi)$ irreducible if $\Phi \neq 0$. If $b_{2}^{+}(\hat{X}) \geq 1$, we can show by the standard argument the following.

Fact B. There is a Baire set of compact supported $C^{r}$ self-dual 2-forms $\eta$ with the following significance.

(1). All the solutions to these equations are irreducible and $\mathcal{M}_{\eta, \mu}(\hat{X}, s)$ is a smooth manifold of dimension given by the index of the linearization of these equations under the gauge fixing condition on the function spaces.

(2). If the dimension of $\mathcal{M}_{\eta, \mu}(\hat{X}, s)$ is negative, then it is empty. 


\subsection{Proof of Proposition 2.2}

Let $X=X_{1} \cup_{Y} X_{2}$ be as in Proposition 2.2. We fix an orientation on $X$ and take the orientation on $Y$ induced so that the orientation on $Y$ preceded by the unit normal vector to $Y$ pointing into $X_{2}$ coincides with that on $X$. We take a collar neighborhood $N$ of $Y$ which is diffeomorphic to $[-1,1] \times Y$. We take a family of metrics $\left\{g_{R}\right\}$ with $R \geq 1$ on $X$, such that they are the same on $X-N$ and $\left.g_{R}\right|_{N}=\lambda_{R}(t) d t^{2}+g_{+}$. Here $d t^{2}$ is the usual flat metric on $[-1,1], \lambda_{R}(t)$ is a positive smooth function on $[-1,1]$ which satisfies

$$
\left\{\begin{array}{l}
\lambda_{R}(t)=1 \text { on }[-1,-1 / 2] \cup[1 / 2,1] \\
\int_{-1 / 2}^{1 / 2} \lambda_{R}(t) d t=R
\end{array}\right.
$$

and $g_{+}$is a metric of positive scalar curvature on $Y$. We denote the Riemanian manifold $\left(X, g_{R}\right)$ by $X_{R}$ and $[-1 / 2,1 / 2] \times Y \subset N$ in $X_{R}$ with the induced metric by $L_{R}$. We put $\hat{X}_{1, R}=X_{1} \cup[-1,0] \times Y$ and $\hat{X}_{2, R}=[0,1] \times Y \cup X_{2}$ with the metrics induced by $g_{R}$. We take a positive smooth function $\beta_{R}$ on $X_{R}$ whose support is contained in $N$, and which is equal to 1 on $L_{R}$ and does not depend on $R$ over $X_{R}-L_{R}$.

Pick a $\operatorname{Spin}^{c}$ structure $s$ on $X$ and we denote the $\operatorname{Spin}^{c}$ structure restricted on $Y$ by $t$. We choose and fix a small exact 2-form $\mu$ on $Y$ for which the unique solution $(\theta, 0)$ on $Y$ is non-degenerate like as in 4.1. Furthermore we choose self-dual 2forms $\eta_{1}$ and $\eta_{2}$ on $X_{R}$ whose supports are contained in $X_{R}-\left(L_{R} \cup X_{2}\right)$ and $X_{R}-$ $\left(L_{R} \cup X_{1}\right)$ respectively, and for which the moduli spaces $\mathcal{M}_{\eta_{1}, \mu}\left(\hat{X}_{1, R}, s \mid \hat{X}_{1, R}\right)$ and $\mathcal{M}_{\eta_{2}, \mu}\left(\hat{X}_{2, R}, s \mid \hat{X}_{2, R}\right)$ consist of irreducible solutions and become smooth manifolds as in 4.2. Here we used the assumptions $b_{2}^{+}\left(X_{i}\right) \geq 1(i=1,2)$. Note that $\eta_{1}$ and $\eta_{2}$ are independent of $R$. Consider the perturbed monopole equations for the $\operatorname{Spin}^{c}$ structure $s$ on $X_{R}$,

$$
\begin{aligned}
\rho\left(F_{A}^{+}+i\left(\eta_{1}+\eta_{2}+\left(\beta_{R} \pi^{*} \mu\right)^{+}\right)\right)-\left\{\Phi \otimes \Phi^{*}\right\} & =0 \\
D_{A}^{+} \Phi & =0 .
\end{aligned}
$$

We write $\mathcal{M}_{\eta_{1}, \eta_{2}, \mu}\left(X_{R}, s\right)$ for the set of gauge equivalence classes of the solutions to these equations. We can choose $\eta_{1}, \eta_{2}$ for which the moduli space $\mathcal{M}_{\eta_{1}, \eta_{2}, \mu}\left(X_{R}, s\right)$ consists of irreducible solutions and is a smooth manifold.

Now suppose that for all sufficiently large $R$, there is an irreducible solution $\left(A_{R}, \Phi_{R}\right)$ in $\mathcal{M}_{\eta_{1}, \eta_{2}, \mu}\left(X_{R}, s\right)$. Then there exists a solution $(A(t), \Phi(t))$ of the equations on $\mathbf{R} \times Y$ which is translation invariant in a temporal gauge.([K-M1]). Here $\mathbf{R} \times Y$ can be regarded as $L_{R}$ with $R \rightarrow \infty$. This solution satisfies the downward gradient flow equations for the functional $C S D_{\mu}$. On the other hand, we have chosen $\mu$ for which the critical point set consists of the non-degenerate single point $(\theta, 0)$. By the standard argument, the uniform bound on $C S D_{\mu}[\mathrm{K}-\mathrm{M} 1]$ and 
the non-degeneracy of the critical point show that there exist limits of $(A(t), \Phi(t))$ as $t \rightarrow \pm \infty$, to which $(A(t), \Phi(t))$ converge with an exponential decay, for taking some gauge transformations. The limits $\lim _{t \rightarrow-\infty}(A(t), \Phi(t))$ and $\lim _{t \rightarrow \infty}(A(t), \Phi(t))$ are both $(\theta, 0)$. Hence this implies that we have elements of $\mathcal{M}_{\eta_{1}, \mu}\left(\hat{X}_{1},\left.s\right|_{\hat{X}_{1}}\right)$ and $\mathcal{M}_{\eta_{2}, \mu}\left(\hat{X}_{2},\left.s\right|_{\hat{X}_{2}}\right)$, where $\hat{X}_{1}=X_{1} \cup[-1, \infty) \times Y$ and $\hat{X}_{2}=(-\infty, 1] \times Y \cup X_{2}$ which can be identified with $\hat{X}_{1, R}$ and $\hat{X}_{2, R}$ respectively as $R \rightarrow \infty$ by certain isometries. Under the identification, we write for $\left.s\right|_{\hat{X}_{i}}$ the $\operatorname{Spin}^{c}$ structures on $\hat{X}_{i}$ induced by $\left.s\right|_{\hat{X}_{i, R}}(i=1,2)$.

On the other hand, the excision property of the indices of the linearization of the equations gives a formula among dimensions

$$
\operatorname{dim} \mathcal{M}_{\eta_{1}, \mu}\left(\hat{X}_{1},\left.s\right|_{\hat{X}_{1}}\right)+\operatorname{dim} \mathcal{M}_{\eta_{2}, \mu}\left(\hat{X}_{2},\left.s\right|_{\hat{X}_{2}}\right)+1=\operatorname{dim} \mathcal{M}_{\eta_{1}, \eta_{2}, \mu}(X, s) .
$$

Here 1 in the left hand side can be interpreted as the dimension of the stabilizer of $(\theta, 0)$.

Now first, we consider the case $\operatorname{dim} \mathcal{M}_{\eta_{1}, \eta_{2}, \mu}(X, s)=0$. The dimension formula tells that one of $\mathcal{M}_{\eta_{1}, \mu}\left(\hat{X}_{1},\left.s\right|_{\hat{X}_{1}}\right)$ and $\mathcal{M}_{\eta_{2}, \mu}\left(\hat{X}_{2},\left.s\right|_{\hat{X}_{2}}\right)$ has negative dimension. But from our choice of $\eta_{1}$ and $\eta_{2}$ in Fact $\mathrm{B}$, it implies one of them is empty. This is a contradiction. Hence we have proved the Seiberg-Witten invariant of $X$ must vanish whenever the moduli space is zero dimensional.

For the case $\operatorname{dim} \mathcal{M}_{\eta_{1}, \eta_{2}, \mu}(X, s)>0$, we can put $\operatorname{dim} \mathcal{M}_{\eta_{1}, \eta_{2}, \mu}(X, s)=2 d$ for some positive integer $d$, because the Seiberg-Witten invariant vanishes if the dimension is odd. We shall use the cut-down argument. (See $[\mathrm{F}],[\mathrm{Sal}]$ ). By the dimension formula, we have either $\operatorname{dim} \mathcal{M}_{\eta_{1}, \mu}\left(\hat{X}_{1},\left.s\right|_{\hat{X}_{1}}\right)<2 d$ or $\mathcal{M}_{\eta_{2}, \mu}\left(\hat{X}_{2},\left.s\right|_{\hat{X}_{2}}\right)<$ $2 d$. Without loss of generality, we may assume $\operatorname{dim} \mathcal{M}_{\eta_{1}, \mu}\left(\hat{X}_{1},\left.s\right|_{\hat{X}_{1}}\right)<2 d$. We take a point $p \in X$ and a small ball $B_{p}$ around $p$. Since the Seiberg-Witten invariant is independent of position of the point (any two points in $X$ are homologically equivalent), it is enough to consider the case $p \in B_{p} \subset X_{1}$. Let $\mathcal{B}_{p}^{*}$ be the set of gauge equivalence classes of pairs $(A, \Phi)$ of spin connections and non-zero spinors over $B_{p}$ for the $\operatorname{Spin}^{c}$ structure $s$. Corresponding to the base point fibration, we have the universal complex line bundle $\mathcal{L}$ over $\mathcal{B}_{p}^{*}$. We can define restriction maps

$$
\begin{gathered}
r_{X}: \mathcal{M}_{\eta_{1}, \eta_{2}, \mu}\left(X_{R}, s\right) \rightarrow \mathcal{B}_{p}^{*} \\
r_{\hat{X}_{1}}: \mathcal{M}_{\eta_{1}, \mu}\left(\hat{X}_{1},\left.s\right|_{\hat{X}_{1}}\right) \rightarrow \mathcal{B}_{p}^{*},
\end{gathered}
$$

because an irreducible solution to the Dirac equation can not vanish on an open set. Then after preparing suitable function space for $\mathcal{B}_{p}^{*}$, we can show, by the usual transversality argument, that there exists a section $\sigma$ of the $d$-fold direct sum $\mathcal{L}^{\oplus d}$ on $\mathcal{B}_{p}^{*}$ such that restriction maps $r_{X}$ and $r_{X_{1}}$ are transverse to $\sigma^{-1}(0)$. We denote by $V \subset \mathcal{B}_{p}^{*}$ the zero set of $\sigma$. The transversality, in particular, implies if the dimension of $\mathcal{M}_{\eta_{1}, \mu}\left(\hat{X}_{1},\left.s\right|_{\hat{X}_{1}}\right) \cap r_{\hat{X}_{1}}^{-1}(V)$ is negative, then it is empty. Now 
by definition, the Seiberg-Witten invariant of $X$ for the $\operatorname{Spin}^{c}$ structure $s$ is the number of points of zero dimensional set $\mathcal{M}_{\eta_{1}, \eta_{2}, \mu}\left(X_{R}, s\right) \cap r_{X}^{-1}(V)$ counted with sign. We suppose that for all sufficiently large $R$ the set $\mathcal{M}_{\eta_{1}, \eta_{2}, \mu}\left(X_{R}, s\right) \cap r_{X}^{-1}(V)$ is nonempty, then the same argument as before shows $\mathcal{M}_{\eta_{1}, \mu}\left(\hat{X}_{1},\left.s\right|_{\hat{X}_{1}}\right) \cap r_{\hat{X}_{1}}^{-1}(V)$ is also nonempty. But the dimension is given by

$$
\operatorname{dim} \mathcal{M}_{\eta_{1}, \mu}\left(\hat{X}_{1},\left.s\right|_{\hat{X}_{1}}\right)-2 d<2 d-2 d=0
$$

This is a contradiction. Hence these moduli spaces are empty. Therefore all the Seiberg-Witten invariants of $X$ must vanish.

Remark. For the case $Y=S^{3} / \Gamma$, we can also prove the vanishing theorem by extending the proof for the case $S^{3}$, (see [Sal], where he uses the removable singularities theorem for the proof), to an equivariant version.

\section{References}

[Ar] V. I. Arnold, Some remarks on symplectic monodromy of Milnor fibrations, "The Floer Memorial Volume", ed. by H. Hofer et al. Progress in Math. 133 Birkhäuser 1995, pp. 99-103.

[Art] M. Artin, Some numerical criteria for contractability of curves on algebraic surfaces, Amer. J. Math. 84 (1962), 485-496.

[B-P-V] W. Barth, C. Peters and A. Van de Ven, Compact complex surfaces, Springer-Verlag, Berlin.

[B1] E. Brieskorn, Über die Aufösung gewisser Singularitäten von holomorphen Abbildungen, Math. Ann. 166 (1966), 76-102.

[B2] E. Brieskorn, Die Auflösung der rationalen Singularitäten holomorpher Abbildungen, Math. Ann. 178 (1968), 255-270.

[B3] E. Brieskorn, Singular elements of semi-simple algebraic groups, Proc. Internat. Congress Math. Nice, 1970, pp. 279-284.

[E] Y. Eliashberg, On Symplectic manifolds with some contact properties, J. Diff. Geom. 33 (1991), 233-238.

[F] K. A. Froyshov, The Seiberg-Witten equations and four-manifolds with boundary, Math. Res. Lett. 3 (1996), 373-390.

[K-O-O] Y. Kanda, H. Ohta and K. Ono, in preparation.

[K-M1] P. B. Kronheimer and T. S. Mrowka, The genus of embedded surfaces in the projective plane, Math. Res. Lett. 1 (1994), 797-808.

[K-M2] P. B. Kronheimer and T. S. Mrowka, Monopoles and contact structures, Invent. Math. 130 (1997), 209-255.

[M1] D. McDuff, Symplectic manifolds with contact type boundaries, Invent. Math. 103 (1991), 651-671.

[M2] D. McDuff, The local behaviour of holomorphic curves in almost complex 4-manifolds, J. Diff. Geom. 34 (1991), 311-358.

[Mi] J. Milnor, Singular points of complex hypersurfaces, Ann. of Math. Studies 61, Princeton Univ. Press. 1968.

[Sai1] K. Saito, Einfach elliptische Singularitaten, Invent. Math. 23 (1974), 289-325.

[Sai2] K. Saito, A new relation among Cartan matrix and Coxeter matrix, J. Algebra 105 (1987), 149-158. 
[Sai3] K. Saito, Around the theory of the generalized weight system: Relations with singularity theory, the generalized Weyl group and its invariant theory, etc., Amer. Math. Soc. Transl. 183 (2) (1998), 101-143.

[Sal] D. Salamon, Removable singularities and a vanishing theorem for Seiberg-Witten invariants, Turkish J. Math. 20 (1996), 61-73.

[T1] C. H. Taubes, The Seiberg-Witten invariants and symplectic forms, Math. Res. Lett. 1 (1994), 809-822.

[T2] C. H. Taubes, The Seiberg-Witten and the Gromov invariants, Math. Res. Lett. 2 (1995), 221-238.

[T3] C. H. Taubes, SW => Gr: From the Seiberg-Witten equations to pseudo-holomorphic curves, J. Amer. Math. Soc. 9 (1996), 845-918.

Added in Proof: In the first paragraph in the section 3, the triviality of the canonical bundle $K_{X}$ implies that $X$ is a spin manifold. Since the Rochlin invariant of $S^{3} / \Gamma$ with $\Gamma=E_{8}$ is non-zero, we have $b_{2}(X) \neq 0$.

Hiroshi Ohta

Graduate School of Mathematics

Nagoya University

Nagoya 464-8602

Japan

e-mail: ohta@math.nagoya-u.ac.jp

(Received: June 9, 1998)
Kaoru Ono

Department of Mathematics

Hokkaido University

Sapporo 060-0810

Japan

e-mail: ono@math.sci.hokudai.ac.jp 